\title{
Bed Planting - A New Crop Establishment Method for Wheat in Rice- Wheat Cropping System
}

\author{
M. I. U. MOLLAH ${ }^{*}$, M. S. U. BHUIYA ${ }^{2} \&$ M. H. KABIR ${ }^{3}$ \\ ${ }^{1}$ Training Division, Bangladesh Rice Research Institute, Gazipur-1701, Bangladesh \\ ${ }^{2}$ Department of Agronomy, Bangladesh Agricultural University, Mymensingh-2202, Bangladesh \\ ${ }^{3}$ Adaptive Research Division, Bangladesh Rice Research Institute, Gazipur-1701, Bangladesh
}

\begin{abstract}
An experiment was conducted at the Bangladesh Rice Research Institute, Gazipur during Rabi season 2001-02 and 2002-03 to determine the effects of bed width, plant row number bed ${ }^{-1}$ and seed rate on the agro-economic productivity of wheat under bed planting in rice-wheat cropping system. Bed planting in 70, 80 and $90 \mathrm{~cm}$ wide beds with two and three plant rows bed ${ }^{-1}$ along with conventional method and 60, 90, 120 and $150 \mathrm{~kg} \mathrm{ha} \mathrm{h}^{-1}$ seed rates were tested. Seventy-centimeter wide beds increased grain yield of wheat up to $21 \%$ over conventional method. It increased the number of panicles $\mathrm{m}^{-2}$, number of grains panicle ${ }^{-1}$ and 1000-grain weight of wheat. Sterility percentage was lower in bed than conventional method. Weed infestation was less in bed planting. It saved 41-48 \% irrigation water. The cost of cultivation was lower and gross return, gross margin and benefit-cost ratio were higher in bed planting than conventional method.
\end{abstract}

Key words: Bed planting, wheat, crop establishment.

\section{INTRODUCTION}

Wheat is grown after rice on the same land in the same year over 26 million ha of South and East Asia to meet the food demand of rapidly expanding human population (Timsina and Connor, 2001). Nearly $60 \%$ of the farming households live on less than $30 \%$ of global agricultural lands (Gupta et al., 2003) and approximately 240 million people in South Asia consume rice and/or wheat produced in rice-wheat system (Benites, 2001). In Bangladesh, rice is the staple food grain and wheat the second important food. The continuous cultivation of rice and wheat, two crops or more year ${ }^{-1}$, has provided food and livelihoods for hundreds of thousands of rural and urban poor in South Asia. To meet the increasing food demand, the productivity of the rice-wheat cropping system must be increased and continued. Development or adoption of new crop establishment methods, changing management practices and inclusion of new crops in the system may be some ways of increasing productivity and resource conservation.

Bed planting in rice-wheat cropping systems may be a technique for improving resource use efficiency and increasing the yield. In this system, the land is prepared conventionally and raised bed and furrows are prepared manually or using a raised bed planting machine. Crops are planted in rows on top of the raised beds and irrigation water is applied in the furrows between the beds.

\footnotetext{
* Corresponding author: PSO, Training Division, BRRI, Gazipur-1701, Mobile: 01712034459. E-mail: miumollah@yahoo.com
}

(C) 2009 School of Agriculture and Rural Development, Bangladesh Open University, All rights reserved. 


\section{I. U. Mollah et al.}

Growing wheat on raised beds though introduced in other countries of the Indo-Gangatic Plain few years ago, in Bangladesh, it is introduced very recently (Connor et al., 2003b). In rice-wheat cropping system, new raised beds are prepared for wheat and after harvesting of wheat, rice is grown in Aman season under zero tillage following a required repairing of the beds.

In conventional system, for transplanting and direct seeding of pre-germinated rice seed, land is prepared by puddling the soil. It causes many problems for following non-rice crops such as destruction of soil structure and enhanced surface cracking. The rice yields were not reduced in non-puddle fields, and yields of wheat after rice on non-puddle fields were higher than the yields of wheat after rice on puddle fields (BRRI, 2000; Hobbs et al., 2002). In bed planting system, as both direct seeding and transplanting are done in zero tillage, no puddling of soil is needed.

Recent research activities in India and Pakistan showed many advantages of bed planting of wheat in rice-wheat systems (Gupta et al., 2000; Hobbs and Gupta, 2003a; Connor et al., 2003a). In Bangladesh, bed planting for rice-wheat systems is very new and research on it is still at introductory phase. Determination of different agronomic aspects of bed planting is essential for development of sustainable rice-wheat cropping system. Therefore, the study was undertaken to determine the effects of bed width, plant row number bed $^{-1}$ and seed rate on the agro-economic productivity of wheat in rice-wheat cropping system.

\section{MATERIALs AND Methods}

The experiment was conducted at the Bangladesh Rice Research Institute (BRRI) experimental farm, Gazipur during Rabi season 2001-02, from November to March and repeated in the next Rabi season 2002-03 on the same plot. The soil of the experimental plot was clay loam with pH 6.78. Bed planting in 70, 80 and $90 \mathrm{~cm}$ (center- to-center of furrows) wide bed with two and three plant rows bed $^{-1}$ along with flat (conventional) planting and 60,90, 120 and $150 \mathrm{~kg}$ seed ha' $\mathrm{a}^{-1}$ were tested. The experiment was laid out in a randomized complete block design with three replications. The unit plot size was $24 \mathrm{~m}^{2}$. Wheat variety Kanchan was used in both the years.

Raised beds and furrows were made manually by spade following the conventional land preparation in the first year. According to the treatments 70,80 and $90 \mathrm{~cm}$ wide beds were made. The height of beds was $15 \mathrm{~cm}$. In the second year, no new beds were made. The beds of the previous year where wheat followed by direct seeded Aman rice were grown kept as permanent bed. It was repaired before seeding of wheat.

Nitrogen, P, K, S and Zn were applied at the rates of 100, 36, 25, 20 and $4 \mathrm{~kg} \mathrm{ha}^{-1}$, respectively. In the first year, two-thirds $\mathrm{N}$ and whole $\mathrm{P}, \mathrm{K}, \mathrm{S}$ and $\mathrm{Zn}$ fertilizers were applied at final land preparation. The remaining one-third $N$ was topdressed at 19 days after sowing at crown root initiation i.e. three leaf stage. This was followed by irrigation. For the treatments with bed planting, $\mathrm{N}$ was applied on the top of beds only. In the second year, the basal doses of fertilizer were applied at final land preparation in the plots with conventional tillage treatment but in the plots with bed planting treatments, the basal doses were applied before sowing on the top of the beds.

Seeds were sown in rows in both bed and conventional methods. For beds, seeds were sown in two and three rows according to the treatments on different width beds. For conventional method, row-to-row distance was $20 \mathrm{~cm}$. In the row seeds were sown continuously and covered with soil properly. The dates of sowing were 29 and 20 November in the first and second year, respectively. Other recommended crop management practices were followed. Weed population and dry biomass of weed were recorded at the time of weeding from a sample area of $0.25 \mathrm{~m}^{2}$. Irrigation water was measured by using a delivery pipe and water pan. Wheat was harvested on 28 and 21 March in the first and second year, respectively. Grain yield and yield components data were collected at maturity. Cost of land preparation, bed preparation and repairing, labour, inputs and irrigation and price of the products and byproducts were recorded. Simple economic analysis such as total variable cost (TVC), gross return, gross margin and benefit-cost ratio (BCR) were done for different methods of planting. 


\section{Results AND Discussion}

\section{Grain yield and yield components}

Effect of different planting methods on grain yield and yield components of wheat were significant for both the years (Table I). The highest grain yield was recorded in $70 \mathrm{~cm}$ wide beds with two plant-rows bed ${ }^{-1}$ (2.85 t ha-1 in 2002 and $3.34 \mathrm{t} \mathrm{ha}^{-1}$ in 2003), which was statistically identical with the grain yield of $70 \mathrm{~cm}$ wide beds with three plant-rows bed ${ }^{-1}\left(2.82 \mathrm{t} \mathrm{ha}^{-1}\right.$ in 2002 and $3.28 \mathrm{t} \mathrm{ha}^{-1}$ in 2003) and significantly higher than conventional method and 80 and $90 \mathrm{~cm}$ wide beds with both two and three plant rows. The yield increase by bed planting using $70 \mathrm{~cm}$ wide beds with two and three plant rows bed ${ }^{-1}$ over conventional method were 21 and $20 \%$, respectively, in 2002 and 19 and $17 \%$, respectively, in 2003 . Similar yield increase by bed planting in wheat was also reported by Dhillon et al. (2000), Gupta et al. (2000), Reeves et al. (2000), Connor et al. (2003b), Hobbs and Gupta (2003b), Sayre (2003), Hossain et al. (2004), Talukder et al. (2004) and Meisner et al. (2005). With the increase in bed width, yield was decreased in both the years. There was no significant yield difference between three and two plant-rows bed ${ }^{-1}$ in same bed width. The highest yield in the bed planting with $70 \mathrm{~cm}$ beds were attributed to higher number of panicles $\mathrm{m}^{-2}$, grains panicle $^{-1}$ and 1000-grain weight.

Table I. Effect of planting method on the yield and yield components of wheat

\begin{tabular}{|c|c|c|c|c|c|c|c|c|c|}
\hline \multicolumn{2}{|c|}{ Method of planting } & \multicolumn{2}{|c|}{$\begin{array}{l}\text { Grain yield } \\
\left(\mathrm{t} \mathrm{ha}^{-1}\right)\end{array}$} & \multicolumn{2}{|c|}{$\begin{array}{l}\text { Panicles } \mathrm{m}^{-2} \\
\text { (no.) }\end{array}$} & \multicolumn{2}{|c|}{$\begin{array}{c}\text { Grains } \\
\text { panicle }^{-1} \text { (no.) }\end{array}$} & \multicolumn{2}{|c|}{ 1000-grain wt. (g) } \\
\hline $\begin{array}{l}\text { Bed width } \\
\text { (cm) }\end{array}$ & $\begin{array}{c}\text { Rows } \\
\text { bed }^{-1} \text { (no.) }\end{array}$ & 2002 & 2003 & 2002 & 2003 & 2002 & 2003 & 2002 & 2003 \\
\hline 70 & 2 & $\begin{array}{l}2.85 a \\
(21)^{\star}\end{array}$ & $\begin{array}{c}3.34 \mathrm{a} \\
(19)\end{array}$ & $306 a$ & $310 a$ & $34.3 \mathrm{a}$ & $36.3 \mathrm{a}$ & $42 . .3 \mathrm{a}$ & $42.3 \mathrm{a}$ \\
\hline 70 & 3 & $\begin{array}{c}2.82 \mathrm{a} \\
(20)\end{array}$ & $\begin{array}{c}3.28 \mathrm{a} \\
(17)\end{array}$ & $312 \mathrm{a}$ & $325 a$ & $32.0 \mathrm{~b}$ & $33.8 \mathrm{~b}$ & $41.7 \mathrm{a}$ & $41.9 \mathrm{a}$ \\
\hline 80 & 2 & $\begin{array}{c}2.54 \mathrm{bc} \\
\text { (8) }\end{array}$ & $\begin{array}{c}2.78 b c \\
(-1)\end{array}$ & $231 \mathrm{c}$ & $260 \mathrm{c}$ & $34.2 \mathrm{a}$ & $35.9 \mathrm{a}$ & $41.3 \mathrm{a}$ & $41.5 \mathrm{a}$ \\
\hline 80 & 3 & $\begin{array}{c}2.65 \mathrm{~b} \\
(13)\end{array}$ & $\begin{array}{c}2.87 \mathrm{~b} \\
(2)\end{array}$ & $244 \mathrm{~b}$ & $282 \mathrm{~b}$ & $31.1 \mathrm{c}$ & $32.9 \mathrm{c}$ & $41.4 \mathrm{a}$ & $41.5 \mathrm{a}$ \\
\hline 90 & 2 & $\begin{array}{c}2.26 \mathrm{~d} \\
(-4)\end{array}$ & $\begin{array}{c}2.64 \mathrm{c} \\
(-6)\end{array}$ & $219 c$ & $241 d$ & $34.2 \mathrm{a}$ & $36.0 \mathrm{a}$ & $41.9 \mathrm{a}$ & $42.1 \mathrm{a}$ \\
\hline 90 & 3 & $\begin{array}{c}2.43 \mathrm{c} \\
(3)\end{array}$ & $\begin{array}{c}2.67 b c \\
(-5)\end{array}$ & $231 \mathrm{c}$ & $242 d$ & $31.3 \mathrm{bc}$ & $33.0 \mathrm{c}$ & $41.5 \mathrm{a}$ & $41.7 \mathrm{a}$ \\
\hline \multicolumn{2}{|c|}{ Conventional } & $2.35 \mathrm{dc}$ & $2.81 \mathrm{bc}$ & $305 a$ & 274 bc & $27.3 d$ & $28.3 d$ & $39.2 \mathrm{~b}$ & $39.6 \mathrm{~b}$ \\
\hline
\end{tabular}

Figures in a column followed by different letters differ significantly at $5 \%$ level of probability as per DMRT. *Figures in the parenthesis are the yield increased (\%) over conventional method.

In first year, conventional method and $70 \mathrm{~cm}$ bed irrespective of plant-rows bed ${ }^{-1}$ produced statistically identical number of panicles $\mathrm{m}^{-2}$ which was significantly higher than other beds. However, in second year, it was higher in $70 \mathrm{~cm}$ wide bed than conventional method. In spite of similar number of panicles $\mathrm{m}^{-2}$ in conventional and $70 \mathrm{~cm}$ bed, the grain yield of conventional method was lower due to lower number of grains panicle ${ }^{-1}$ and lower grain weight. Bed planting method produced significantly higher grains panicle ${ }^{-1}$ than conventional method. The number of grains panicle ${ }^{-1}$ was also significantly higher in two plant rows bed ${ }^{-1}$ than three plant-rows. Weight of 1000-grain was also significantly higher in bed planting than conventional method. Yadav et al. (2002), Zhongming and Fahong (2005) and Meisner et al. (2005) reported similar results.

Seed rate did not show any significant effect on grain yield and yield components in both the years. Though $120 \mathrm{~kg} \mathrm{ha}^{-1}$ seed rate gave the highest yield and $60 \mathrm{~kg} \mathrm{ha}^{-1}$ the lowest yield, it was insignificant (Table II). No significant interaction effect of planting method and seed rate on grain yield and yield components were observed in both the years. 
Table II. Effect of seed rate on the yield and yield components of wheat

\begin{tabular}{ccccccccc}
\hline \multirow{2}{*}{$\begin{array}{c}\text { Seed rate } \\
\left(\mathrm{kg} \mathrm{ha}^{-1}\right)\end{array}$} & \multicolumn{2}{c}{$\begin{array}{c}\text { Grain yield } \\
\left(\mathrm{t} \mathrm{ha}^{-1}\right)\end{array}$} & \multicolumn{2}{c}{$\begin{array}{c}\text { Panicles } \mathrm{m}^{-2} \\
(\text { no. })\end{array}$} & \multicolumn{2}{c}{$\begin{array}{c}\text { Grains } \\
\text { panicle }\end{array}$} & \multicolumn{2}{c}{$\begin{array}{c}\text { 1000-grain wt. } \\
(\mathrm{g})\end{array}$} \\
\cline { 2 - 10 } & 2002 & 2003 & 2002 & 2003 & 2002 & 2003 & 2002 & 2003 \\
\hline 150 & 2.62 & 2.95 & 268 & 283 & 32.3 & 34.0 & 41.4 & 41.3 \\
120 & 2.68 & 2.98 & 268 & 279 & 31.9 & 33.8 & 41.6 & 42.0 \\
90 & 2.55 & 2.89 & 261 & 274 & 32.0 & 33.7 & 41.2 & 41.5 \\
60 & 2.49 & 2.82 & 259 & 268 & 32.0 & 33.6 & 41.1 & 41.3 \\
\hline
\end{tabular}

\section{Other plant attributes}

Planting method significantly affected plant height, panicle length, non-bearing tillers $\mathrm{m}^{-2}$, sterility percentage, straw yield and harvest index of wheat. Plant height, panicle length and harvest index were higher in bed planting. On the contrary, non-bearing tillers $\mathrm{m}^{-2}$ and sterility percentage were higher in conventional method (Table III). However, straw yield was similar in $70 \mathrm{~cm}$ wide bed and conventional method. Seventy-centimeter beds with two and three plant-rows bed ${ }^{-1}$ produced longer panicles (13.9 and $13.7 \mathrm{~cm}$ in 2002, and 14.3 and $14.1 \mathrm{~cm}$ in 2003). Significantly lower number of non-bearing tillers $\mathrm{m}^{-2}$ was recorded in all bed planting treatments than conventional method. Bed planting significantly reduced the sterility percentage compared to conventional method. In bed planting, sterility was lower in beds with two plant-rows than three plant-rows irrespective of bed width and it was also lower in narrow beds than wider beds. The lower sterility might be accountable for higher grains in bed planting. Bed planting resulted in higher harvest index than conventional method.

Table III. Effect of planting method on different growth parameters of wheat

\begin{tabular}{|c|c|c|c|c|c|c|c|}
\hline \multicolumn{2}{|c|}{ Method of planting } & \multirow{2}{*}{$\begin{array}{l}\text { Plant } \\
\text { height } \\
(\mathrm{cm})\end{array}$} & \multirow{2}{*}{$\begin{array}{c}\text { Panicle } \\
\text { length }(\mathrm{cm})\end{array}$} & \multirow{2}{*}{$\begin{array}{c}\text { Non-bearing } \\
\text { tillers } \\
\text { (no. } \mathrm{m}^{-2} \text { ) } \\
\end{array}$} & \multirow[b]{2}{*}{$\begin{array}{c}\text { Sterility } \\
(\%)\end{array}$} & \multirow[b]{2}{*}{$\begin{array}{l}\text { Straw yield } \\
\left(\mathrm{t} \mathrm{ha}^{-1}\right)\end{array}$} & \multirow[b]{2}{*}{$\begin{array}{l}\text { Harvest } \\
\text { index }\end{array}$} \\
\hline $\begin{array}{l}\text { Bed width } \\
(\mathrm{cm})\end{array}$ & $\begin{array}{c}\text { Rows bed } \\
\text { (no.) }\end{array}$ & & & & & & \\
\hline & & & & 2002 & & & \\
\hline 70 & 2 & $97.3 \mathrm{a}$ & $13.9 \mathrm{a}$ & $3.5 \mathrm{c}$ & $11.9 \mathrm{c}$ & $5.44 \mathrm{bc}$ & $0.34 \mathrm{a}$ \\
\hline 70 & 3 & $97.3 \mathrm{a}$ & $13.7 \mathrm{a}$ & $5.6 \mathrm{~b}$ & $14.9 \mathrm{~b}$ & $6.11 \mathrm{a}$ & $0.30 \mathrm{c}$ \\
\hline 80 & 2 & $96.8 \mathrm{a}$ & $13.4 \mathrm{ab}$ & $3.2 \mathrm{c}$ & $12.9 \mathrm{c}$ & $4.78 \mathrm{~d}$ & $0.35 \mathrm{a}$ \\
\hline 80 & 3 & $97.0 \mathrm{a}$ & $13.2 \mathrm{ab}$ & $3.8 \mathrm{c}$ & $16.0 \mathrm{~b}$ & $5.41 \mathrm{c}$ & $0.29 \mathrm{~d}$ \\
\hline 90 & 2 & $97.2 \mathrm{a}$ & $12.6 \mathrm{bc}$ & $4.0 \mathrm{c}$ & $13.3 \mathrm{c}$ & $4.90 \mathrm{~d}$ & $0.31 \mathrm{~b}$ \\
\hline 90 & 3 & $97.3 \mathrm{a}$ & $12.5 \mathrm{bc}$ & $4.0 \mathrm{c}$ & $16.0 \mathrm{~b}$ & $5.65 \mathrm{~b}$ & $0.30 \mathrm{c}$ \\
\hline \multicolumn{2}{|c|}{ Conventional } & $85.1 \mathrm{~b}$ & $12.0 \mathrm{c}$ & $\begin{array}{c}10.8 \mathrm{a} \\
2003\end{array}$ & $20.5 \mathrm{a}$ & $5.46 \mathrm{bc}$ & $0.30 \mathrm{c}$ \\
\hline 70 & 2 & $99.9 \mathrm{~b}$ & $14.3 \mathrm{a}$ & $1.8 \mathrm{~cd}$ & $11.5 \mathrm{~d}$ & $6.02 \mathrm{~b}$ & $0.36 \mathrm{a}$ \\
\hline 70 & 3 & $101.2 \mathrm{ab}$ & $14.1 \mathrm{a}$ & $2.5 \mathrm{~b}$ & 14.6 bc & $6.44 \mathrm{a}$ & $0.34 \mathrm{~b}$ \\
\hline 80 & 2 & $102.3 \mathrm{a}$ & $13.4 \mathrm{ab}$ & $3.2 \mathrm{~b}$ & $12.6 \mathrm{~d}$ & $5.44 \mathrm{~d}$ & $0.34 \mathrm{~b}$ \\
\hline 80 & 3 & $102.4 \mathrm{a}$ & $13.3 \mathrm{ab}$ & $2.7 \mathrm{~b}$ & 15.5 b & $5.77 \mathrm{c}$ & $0.33 \mathrm{c}$ \\
\hline 90 & 2 & $102.2 \mathrm{a}$ & $13.1 \mathrm{ab}$ & $1.9 \mathrm{~cd}$ & $13.0 \mathrm{~cd}$ & $5.20 \mathrm{e}$ & $0.34 \mathrm{~b}$ \\
\hline 90 & 3 & 101.8 a & $12.8 \mathrm{~b}$ & $1.5 \mathrm{~d}$ & 15.9 b & $5.54 \mathrm{~d}$ & $0.33 \mathrm{c}$ \\
\hline \multicolumn{2}{|c|}{ Conventional } & $91.9 \mathrm{c}$ & $12.3 \mathrm{~b}$ & $6.2 \mathrm{a}$ & $19.2 \mathrm{a}$ & $6.35 \mathrm{a}$ & $0.31 \mathrm{~d}$ \\
\hline
\end{tabular}

Figures in a column followed by different letters differ significantly at $5 \%$ level of probability as per DMRT.

Seed rate did not have significant effect on plant height, panicle length, number of non-bearing tillers, sterility and harvest index of wheat. However, higher seed rate produced significantly higher straw yield in both the years (Table IV). 
Table IV. Effect of seed rate on different growth parameters of wheat

\begin{tabular}{ccccccc}
\hline $\begin{array}{c}\text { Seed rate } \\
\left(\mathrm{kg} \mathrm{ha}^{-2}\right)\end{array}$ & $\begin{array}{c}\text { Plant height } \\
(\mathrm{cm})\end{array}$ & $\begin{array}{c}\text { Panicle length } \\
(\mathrm{cm})\end{array}$ & $\begin{array}{c}\text { Non-bearing } \\
\text { tillers }\left(\mathrm{no.}^{-2}\right)\end{array}$ & $\begin{array}{c}\text { Sterility } \\
(\%)\end{array}$ & $\begin{array}{c}\text { Straw yield } \\
\left(\mathrm{t} \mathrm{ha}^{-1}\right)\end{array}$ & $\begin{array}{c}\text { Harvest } \\
\text { index }\end{array}$ \\
\hline & & & 2002 & & & \\
150 & 96.0 & 13.1 & 4.6 & 14.6 & $5.50 \mathrm{a}$ & 0.31 \\
120 & 95.1 & 13.1 & 4.7 & 14.9 & $5.51 \mathrm{a}$ & 0.32 \\
90 & 95.3 & 13.0 & 5.1 & 15.3 & $5.29 \mathrm{~b}$ & 0.32 \\
60 & 95.3 & 13.0 & 5.4 & 15.5 & $5.28 \mathrm{~b}$ & 0.31 \\
& & & 2003 & & & \\
150 & 100.1 & 13.3 & 2.7 & 14.2 & $6.06 \mathrm{a}$ & 0.33 \\
120 & 100.5 & 13.4 & 3.6 & 14.5 & $5.87 \mathrm{~b}$ & 0.34 \\
90 & 100.0 & 13.3 & 2.1 & 14.8 & $5.72 \mathrm{c}$ & 0.34 \\
60 & 100.3 & 13.3 & 2.7 & 15.0 & $5.64 \mathrm{c}$ & 0.33 \\
\hline
\end{tabular}

Figures in a column followed by different letters differ significantly at $5 \%$ level of probability as per DMRT.

\section{Weed population}

Weed population and dry biomass were greatly influenced by different planting methods of wheat. Bed planting significantly reduced weed population resulting in lower dry biomass than conventional method in both the new and old beds. The lowest number of weeds $\mathrm{m}^{-2}$ and dry biomass yield were recorded in the $70 \mathrm{~cm}$ wide beds with three plant rows bed ${ }^{-1}$ which was followed by same width bed with two plant rows bed ${ }^{-1}$ (Table V). Ram et al. (2005) also found lower weed biomass in raised beds than the conventional method. Both weed population and dry biomass yield were increased with the increase in width of beds and these were also higher in bed with two plant rows than three plant rows. The low number of weeds in beds might be due to dry top surface of beds that inhibited the weed growth. Moreover, at the time of bed preparation, the top soils of the furrows were mulched to the raised beds, which drastically reduced the weeds in furrows.

Table V. Weed vegetation in wheat as influenced by method of planting

\begin{tabular}{|c|c|c|c|c|c|}
\hline \multicolumn{2}{|c|}{ Method of planting } & \multicolumn{4}{|c|}{ Weed vegetation } \\
\hline \multirow[b]{2}{*}{ Bed width $(\mathrm{cm})$} & \multirow[b]{2}{*}{ Rows bed ${ }^{-1}$ (no.) } & \multicolumn{2}{|c|}{2002} & \multicolumn{2}{|c|}{2003} \\
\hline & & $\begin{array}{l}\text { Population } \\
\text { (no. } \mathrm{m}^{-2} \text { ) }\end{array}$ & $\begin{array}{l}\text { Dry biomass } \\
\left(\mathrm{kg} \mathrm{ha}^{-1}\right)\end{array}$ & $\begin{array}{l}\text { Population } \\
\text { (no. } \mathrm{m}^{-2} \text { ) }\end{array}$ & $\begin{array}{c}\text { Dry biomass } \\
\left(\mathrm{kg} \mathrm{ha}^{-1}\right)\end{array}$ \\
\hline 70 & 2 & $64 \mathrm{f}$ & $55.7 \mathrm{e}$ & $77 \mathrm{f}$ & $69.6 \mathrm{f}$ \\
\hline 70 & 3 & $51 \mathrm{~g}$ & $47.2 \mathrm{f}$ & $59 \mathrm{~g}$ & $53.5 \mathrm{~g}$ \\
\hline 80 & 2 & $105 \mathrm{~d}$ & $96.7 \mathrm{c}$ & $120 \mathrm{~d}$ & $104.5 \mathrm{~d}$ \\
\hline 80 & 3 & $83 \mathrm{e}$ & $71.2 \mathrm{~d}$ & $96 \mathrm{e}$ & $85.5 \mathrm{e}$ \\
\hline 90 & 2 & $136 \mathrm{~b}$ & $115.0 \mathrm{~b}$ & $162 \mathrm{~b}$ & $147.4 \mathrm{~b}$ \\
\hline 90 & 3 & $116 \mathrm{c}$ & $97.4 \mathrm{c}$ & $136 \mathrm{c}$ & $123.5 \mathrm{c}$ \\
\hline \multicolumn{2}{|c|}{ Conventional } & $205 a$ & $173.2 \mathrm{a}$ & $240 a$ & $207.8 \mathrm{a}$ \\
\hline
\end{tabular}

Figures in a column followed by different letters differ significantly at $5 \%$ level of probability as per DMRT.

Seed rate also significantly affected the weed population and dry biomass yield. The lowest number of weeds $\mathrm{m}^{-2}$ and dry biomass yield were recorded with $150 \mathrm{~kg} \mathrm{ha}^{-1}$ seed rate. With the decrease in seed rate, both the weed population and dry biomass yield were increased (Table $\mathrm{VI})$. Cyperus rotundus, Cynodon dactylon, Echinochloa colonum, Euphorbia hirta, Vicia hirsuta and Vicia sativa were the major weeds in wheat in both the years for all the planting methods.

\section{Irrigation water}

Amount of water required for different irrigations differed remarkably between the conventional and bed planting methods. The conventional method received the highest amount of water at every 
irrigation and total amount was $315 \mathrm{~mm}$ and $318 \mathrm{~mm}$ in 2001-02 and 2002-03, respectively (Table VII). Total water savings by 70,80 and $90 \mathrm{~cm}$ wide beds over conventional method were $41-46 \%$, $42-48 \%$ and $44-48 \%$, respectively. Among the beds, the narrow bed $(70 \mathrm{~cm})$ required slightly higher amount of irrigation water than wider bed. In the bed planting, irrigation water was applied only in furrows. The area of furrows unit ${ }^{-1}$ area in the wider beds is lower than the narrow beds. So, it received lower amount of irrigation water. Savings of irrigation water by bed planting of wheat ranged from $18 \%$ to $50 \%$ were reported many scientists (Gupta et al., 2000; Yadav et al., 2002; Gupta, 2003; Hobbs and Gupta, 2003b and Sayre, 2003).

Table VI. Weed vegetation in wheat as influenced by seed rate of wheat

\begin{tabular}{clccc}
\hline \multirow{2}{*}{$\begin{array}{c}\text { Seed rate } \\
\left(\mathrm{kg} \mathrm{ha}^{-1}\right)\end{array}$} & \multicolumn{4}{c}{ Weed vegetation } \\
\cline { 2 - 5 } & \multicolumn{1}{c}{$\begin{array}{c}\text { Population } \\
\left(\text { no. } \mathrm{m}^{-2}\right)\end{array}$} & $\begin{array}{c}\text { Dry biomass } \\
\left(\mathrm{kg} \mathrm{ha}^{-1}\right)\end{array}$ & $\begin{array}{c}\text { Population } \\
\left(\text { (no. m}^{-2}\right)\end{array}$ & $\begin{array}{c}\text { Dry biomass } \\
\left(\mathrm{kg} \mathrm{ha}^{-1}\right)\end{array}$ \\
\hline 150 & $101 \mathrm{c}$ & $86.8 \mathrm{c}$ & $118 \mathrm{c}$ & $103.0 \mathrm{c}$ \\
120 & $106 \mathrm{bc}$ & $92.5 \mathrm{~b}$ & $126 \mathrm{~b}$ & $111.1 \mathrm{~b}$ \\
90 & $111 \mathrm{ab}$ & $96.0 \mathrm{a}$ & $131 \mathrm{a}$ & $117.2 \mathrm{ab}$ \\
60 & $115 \mathrm{a}$ & $99.8 \mathrm{a}$ & $134 \mathrm{a}$ & $121.1 \mathrm{a}$ \\
\hline
\end{tabular}

Figures in a column followed by different letters differ significantly at $5 \%$ level of probability as per DMRT.

Table VII. Irrigation water savings by bed planting of wheat over conventional method

\begin{tabular}{|c|c|c|c|c|c|c|}
\hline \multirow[b]{2}{*}{ Tillage option } & \multicolumn{5}{|c|}{ Water required at different times of irrigation $(\mathrm{mm})$} & \multirow{2}{*}{$\begin{array}{c}\text { Water saved } \\
\text { over } \\
\text { conventional (\%) }\end{array}$} \\
\hline & Sowing & $\begin{array}{l}\text { Crown root } \\
\text { initiation }\end{array}$ & $\begin{array}{l}\text { Maximum } \\
\text { tillering }\end{array}$ & Grain filling & Total & \\
\hline & & & $2001-02$ & & & \\
\hline $70 \mathrm{~cm}$ bed & 57 & 49 & 41 & 23 & 170 & 46 \\
\hline $80 \mathrm{~cm}$ bed & 55 & 49 & 40 & 21 & 165 & 48 \\
\hline $90 \mathrm{~cm}$ bed & 55 & 48 & 39 & 21 & 163 & 48 \\
\hline Conventional & 95 & 89 & $\begin{array}{c}76 \\
2002-03\end{array}$ & 55 & 315 & - \\
\hline $70 \mathrm{~cm}$ bed & 58 & 48 & 45 & 35 & 186 & 41 \\
\hline $80 \mathrm{~cm}$ bed & 56 & 46 & 44 & 34 & 180 & 42 \\
\hline $90 \mathrm{~cm}$ bed & 55 & 45 & 42 & 32 & 174 & 44 \\
\hline Conventional & 94 & 85 & 79 & 60 & 318 & - \\
\hline
\end{tabular}

\section{Economic analysis}

A simple economic analysis such as total variable cost (TVC), gross return, gross margin and benefit-cost ratio (BCR) for wheat for different methods of planting are shown in Table VIII. The TVC of conventional method (TK.16510 ha ${ }^{-1}$ in 2002 and TK.16690 ha ${ }^{-1}$ in 2003) was higher than bed planting in 70, 80 and $90 \mathrm{~cm}$ wide beds with the both two and three plant rows. Though in bed planting system an additional cost of bed preparation for the first year was included in the TVC, the cost of irrigation water and labour costs of sowing, weeding and harvesting were lower than conventional method. Many researchers also reported lower costs of production in bed planting which ranged from 20-30 \% compared to conventional method (Reeves et al., 2000; Sayre, 2003 and Connor et al., 2003b). In the second year, the TVC of bed planting was much lower since wheat was grown on the same beds prepared in the first year. No land preparation cost was needed and bed repairing cost was added instead of bed preparation cost. Rautaray (2004) showed similar results. The cost of weeding was lower in bed planting than conventional method because of less weed population. 
Bed Planting - A New Crop Establishment Method for Wheat in Rice-Wheat Cropping System

Table VIII. Agro-economic productivity of wheat as affected by method of planting

\begin{tabular}{|c|c|c|c|c|c|c|c|}
\hline \multicolumn{2}{|c|}{ Method of planting } & \multicolumn{2}{|c|}{ Yield $\left(\mathrm{t} \mathrm{ha}^{-1}\right)$} & \multirow{2}{*}{$\begin{array}{c}\text { Total } \\
\text { variable cost } \\
\left(000^{\prime} \mathrm{Tk} \mathrm{ha}^{-1}\right)\end{array}$} & \multirow[b]{2}{*}{$\begin{array}{l}\text { Gross return } \\
\left(000 \mathrm{Tk} \mathrm{ha}^{-1}\right)\end{array}$} & \multirow[b]{2}{*}{$\begin{array}{l}\text { Gross margin } \\
\left(000^{\prime} \mathrm{Tk}^{-1}\right)\end{array}$} & \multirow[b]{2}{*}{ BCR } \\
\hline $\begin{array}{l}\text { Bed width } \\
(\mathrm{cm})\end{array}$ & $\begin{array}{l}\text { Rows bed }{ }^{-1} \\
\text { (no.) }\end{array}$ & Grain & Straw & & & & \\
\hline & & & & 2002 & & & \\
\hline 70 & 2 & 2.85 & 5.44 & 15.81 & 31.09 & 15.28 & 1.97 \\
\hline 70 & 3 & 2.82 & 6.11 & 16.23 & 31.49 & 15.26 & 1.94 \\
\hline 80 & 2 & 2.54 & 4.78 & 15.45 & 27.64 & 12.19 & 1.79 \\
\hline 80 & 3 & 2.65 & 5.41 & 15.76 & 29.26 & 13.50 & 1.86 \\
\hline 90 & 2 & 2.26 & 4.90 & 15.12 & 25.24 & 10.12 & 1.67 \\
\hline 90 & 3 & 2.43 & 5.65 & 15.50 & 27.52 & 12.02 & 1.78 \\
\hline \multicolumn{2}{|c|}{ Conventional } & 2.35 & 5.46 & 16.51 & 26.61 & 10.10 & 1.61 \\
\hline 70 & 2 & 3.34 & 6.02 & 13.52 & 36.08 & 22.56 & 2.67 \\
\hline 70 & 3 & 3.28 & 6.44 & 13.80 & 35.96 & 22.16 & 2.61 \\
\hline 80 & 2 & 2.78 & 5.44 & 13.13 & 30.46 & 17.33 & 2.32 \\
\hline 80 & 3 & 2.87 & 5.77 & 13.45 & 31.60 & 18.15 & 2.35 \\
\hline 90 & 2 & 2.64 & 5.20 & 12.94 & 28.96 & 16.02 & 2.24 \\
\hline 90 & 3 & 2.67 & 5.54 & 13.32 & 29.57 & 16.25 & 2.22 \\
\hline \multicolumn{2}{|c|}{ Conventional } & 2.81 & 6.35 & 16.69 & 31.64 & 14.95 & 1.90 \\
\hline
\end{tabular}

Local market price: Wheat $=9.00 \mathrm{Tk} \mathrm{kg}^{-1}$ and wheat straw $=1.00 \mathrm{Tk} \mathrm{kg}^{-1}$, wheat seed $=15.00 \mathrm{Tk} \mathrm{kg}^{-1}$.

Labour wages: Tk 8.75 man-hour ${ }^{-1}$. Fertilizer cost: Urea $=6.00 \mathrm{Tk}^{\mathrm{kg}}{ }^{-1}, \mathrm{TSP}=12.00 \mathrm{Tk}^{\mathrm{kg}} \mathrm{kg}^{-1}, \mathrm{MP}=10.00 \mathrm{Tk}$. $\mathrm{kg}^{-1}$, Gypsum $=4.00 \mathrm{Tk} \cdot \mathrm{kg}^{-1}$ and $\mathrm{ZnSO}_{4}=40.00 \mathrm{Tk} \cdot \mathrm{kg}^{-1}$.

In the first year, $70 \mathrm{~cm}$ wide bed with three plant rows gave the highest gross return (Tk 31490 $\mathrm{ha}^{-1}$ ) which was followed by $70 \mathrm{~cm}$ wide bed with two plant rows (Tk $31090 \mathrm{ha}^{-1}$ ). Whereas, in the following year, $70 \mathrm{~cm}$ wide bed with two plant rows gave the highest gross return (Tk $36080 \mathrm{ha}^{-1}$ ) which was followed by $70 \mathrm{~cm}$ wide bed with three plant rows $\left(\mathrm{Tk} 35960 \mathrm{ha}^{-1}\right)$. The highest gross margin (Tk.15,280 ha ${ }^{-1}$ and Tk.22,560 ha has $^{-1}$ obtained in $70 \mathrm{~cm}$ wide bed with two plant rows which was followed by $70 \mathrm{~cm}$ bed with three plant rows (Tk.15,260 ha ${ }^{-1}$ and Tk.22,160 ha ${ }^{-1}$ ). With the increase in width of beds, the gross margins were decreased and it was the least in conventional method. The BCR followed the same pattern as observed in gross margin. The highest BCR was recorded in bed planting in $70 \mathrm{~cm}$ wide beds with two plant rows (1.97 and 2.67) which were followed by $70 \mathrm{~cm}$ wide bed with three rows $(1.94$ and 2.61 and it was the lowest in conventional method (1.61 and 1.90). Hassan et al. (2005) also found higher net benefit and BCR in the bed planting compared to the conventional method.

\section{CONCLUSION}

From the two years results it may be concluded that wheat could be grown successfully on raised bed and agro-economic productivity of wheat in rice-wheat cropping system could be increased by bed planting with $70 \mathrm{~cm}$ width of bed over conventional method.

\section{Literature Cited}

Benites, J. R. 2001. Socio-economic perspectives, policy implications and food security for conservation agriculture in Asia. Presented in Intl. Workshop on Conservation Agriculture for Food Security and Environment Protection in Rice-Wheat Cropping Systems. 6-9 Feb. 2001. Lahore, Pakistan.

BRRI (Bangladesh Rice Research Institute). 2000. In: BRRI Annual Research Review for 1999. XIX. Rice Farming Systems. October 9-12, 2000. Bangladesh Rice Res. Inst. Gazipur, Bangladesh. 
Connor, D. J., Timsina, J., and Humphreys, E. 2003a. Prospects for permanent beds for the ricewheat system. In "Improving Productivity and Sustainability of Rice-Wheat Systems: Issues and Impact". American Soc. Agron. Spec. Publ. 65, 197-210.

Connor, D. J., Gupta, R. K., Hobbs, P. R. and Sayre, K. D. 2003b. Bed planting in rice-wheat systems. In: Addressing Resource Conservation Issues in Rice-Wheat Systems of South Asia: A Resource Book. Rice-Wheat Consortium for the Indo-Gangetic Plains. Intl. Maize and Wheat Impr. Cent., New Delhi, India. pp. 103-108.

Dhillon, S. S., Hobbs, P. R. and Samra, J.S. 2000. Investigations on bed planting system as an alternative tillage and crop establishment practice for improving wheat yields sustainability. In: Proc. 15th Conf. of the Intl. Soil Tillage Res. Org. 2-7 July 2000. Fort Worth, Texas, USA.

Gupta, R. K. 2003. Is conventional tillage essential for wheat? In: Addressing Resource Conservation Issues in Rice-Wheat Systems of South Asia: A Resource Book. Rice-Wheat Consortium for the Indo-Gangetic Plains. Intl. Maize and Wheat Impr. Cent., New Delhi, India. pp. 95-100.

Gupta, R. K., Hobbs, P. R., Salim, M., Malik, R. K., Varma, M. R., Pokharel, T. P., Thakur, T. C. and Tripathri, J. 2000. Research and extension issues for farm-level impact on the productivity on rice-wheat systems in the Indo-Gangetic Plains of India and Pakistan. Rice-Wheat Consortium Sem. Rep. Ser. 1. Rice-Wheat Consortium for the Indo-Gangetic Plains. New Delhi, India. 26 p.

Gupta, R. K., Listman, G. M. and Harrington, L. 2003. The Rice-Wheat Consortium for the IndoGangetic Plains: Vision and management structure. In: Addressing Resource Conservation Issues in Rice-Wheat Systems of South Asia: A Resource Book. Rice-Wheat Consortium for the Indo-Gangetic Plains. Intl. Maize and Wheat Impr. Cent., New Delhi, India. pp. 1-7.

Hassan, I., Hussain, Z. and Akbar, G. 2005. Effect of permanent raised beds on productivity of water use for irrigated maize-wheat cropping system. Presented in ACIAR Workshop on Permanent Bed Planting Systems, 1-3 Mar. 2005. Griffith, NSW, Australia.

Hobbs, P. R. and Gupta, R. K. 2003a. Resource-conserving technologies for wheat in the ricewheat system. In: Improving Productivity and Sustainability of Rice-Wheat Systems: Issues and Impact. American Soc. Agron. Spec. Publ. 65, 149-171.

Hobbs, P. R. and Gupta, R. K. 2003b. Rice-wheat cropping systems in Indo-Gangetic Plains: Issues of Water Productivity in Relation to New Resource-Conserving Technologies. In: Kijne et al. (ed.) Water Productivity in Agriculture: Limits and Opportunities for Improvement. pp. 239-253.

Hobbs, P. R., Singh, Y., Giri, G. S., Lauren, J. G. and Duxbury, J. M. 2002. Direct-seeding and reduced-tillage options in the rice-wheat systems of the Indo-Gangetic Plains of South Asia. In: Pandey et al. (ed.) Direct seeding: Research issues and opportunities. Proc. Intl. Workshop on Direct Seeding in Asian Rice Systems: Strategic Research Issues and Opportunities. Bangkok, Thailand. 25-28 Jan. 2002. Intl. Rice Res. Inst., Los Banos, Philippines. pp. 201-215.

Hossain, M. I., Meisner, C., Duxbury, J. M., Lauren, J. G, Rahman, M. M., Meer, M. M. and Rashid, M. H. 2004. Use of raised beds for increasing wheat production in rice-wheat cropping systems. New directions for a diverse planet. Presented in 4th Intl. Crop Sci. Cong. 26 Sep. 1 Oct. 2004. Brisbane, Australia.

Meisner, C. A., Talukdar, H. M., Hossain, I., Gill, M., Rahmen, H. M., Baksh, E., Justice, S. and Sayre, K. D.2005. Introduction and implementing a permanent bed system in the rice-wheat cropping pattern in Bangladesh and Pakistan. Presented in ACIAR Workshop on Permanent Bed Planting Systems. 1-3 Mar. 2005. Griffith, NSW, Australia.

Ram, H., Singh, Y. Kler, D. S., Kumar, K., Humphreys, L. and Timsina, J. 2005. Performance of non-rice crops and alternative cropping systems on permanent raised beds in the Indo- 
Gangetic plains of north-western India. Presented in ACIAR Workshop on Permanent Bed Planting Systems. 1-3 Mar. 2005. Griffith, NSW, Australia.

Rautaray, S. K. 2004. Raised bed planting of wheat. In: Nat. Conf. on Conservation Agriculture: Conserving Resourses-Enhanching Productivity. 22-23 Sep. 2004. NASC Complex, Dev Prakash Shastri Marg, Pusa, New Delhi 110012. Abst. pp. 17-18.

Reeves, T. G., Ransom, J. K., Sayre, K. D., Hobbs, P. R. and Cassaday, K. 2000. From Scarcity to Security: A Global Perspective on Weed Science and the Future Food Situation. Presented in 3rd Weed Sci. Cong. on Global Weed Problems: Local and Global Solutions for the Beginning of the Century. 6-11 Jun. 2000, Foz de Iguacu, Brazil.

Sayre, K. D. 2003. Raised bed system of cultivation. In: Bed Planting Training Course. 19 May -21 Jun. 2003. Intl. Maize and Wheat Impr. Cent. Mexico.

Talukder, A. S. M. H. M., Meisner, C.A., Kabir, M. J., Hossain, A. B. S. and Rashid, M. H. 2004. Productivity of multi-crops sown on permanent raised beds in the tropics. New directions for a diverse planet: Presented in 4th Intl. Crop Sci. Cong. 26 Sep.-1Oct. 2004. Brisbane, Australia.

Timsina, J. and Connor, D. J. 2001. Productivity and management of rice-wheat cropping systems: issues and challenges. Field Crop Res. 69, 93-132.

Yadav, A., Malik, R. K., Chouhan, B. S., Kumar, V., Banga, P. S., Singh, S., Yadav, J. S., Punia, S. S., Rathee, S. S. and Sayre, K. D. 2002. Feasibility of raising wheat on furrow irrigated raised beds in South-Western Haryana. Presented in Intl. workshop on, Herbicide resistance management and zero tillage in rice-what cropping system. 4-6 Mar 2002, Hisar, Haryana, India.

Zhongming, M. and Fahong, W. 2005. Raised bed-planting system for irrigated spring wheat in Hexi Corridor. Presented in ACIAR Workshop on Permanent Bed Planting Systems. 1-3 March 2005, Griffith, NSW, Australia. 